\title{
Health Care for Elderly
}

\author{
Siniša Franjic ${ }^{1 *}$ \\ ${ }^{1}$ Independent Researcher \\ *Corresponding Author \\ Siniša Franjić
}

\section{Article History}

Received: 27.02.2021

Accepted: 30.03 .2021

Published: 03.04.2021

\begin{abstract}
Health care is a system of state, group and individual measures for the improvement, preservation and restoration of health. The goal of health care is the promotion, ie improvement of health, prevention, ie prevention of diseases, timely detection of diseases, effective treatment and rehabilitation. Health care is organized on three levels, so it is about primary, secondary and tertiary health care. In cases of justified emergencies, every person has the right to emergency medical care and emergency treatment, regardless of legal status. In the event that a large number of persons whose medical condition requires urgent medical care is present in one place, triage is carried out in order to determine the cases according to the degree of urgency and to give priority to the most urgent ones.
\end{abstract}

Keywords: Elderly, Health, Health Care, Disease, Insurance, Prevention.

\section{INTRODUCTION}

Despite considerable changes in family forms during the past decades, the influence of family on health is strong and persistent [1]. All over Europe the elderly still live in more traditional family forms related to marriage and their family biographies are closely tied to the civil status of their partnership. On the other hand, new family forms have been emerging among the young, yet the prevalence and acceptance of these forms differs widely between societies. At a young age, the distribution of paid and unpaid work within the household is largely gendered, and in the case of families with children it is centred around the care provision for children; at old age, when paid work has ceased and income is secured by pension systems, the distribution of unpaid work is less of an issue, while the care provision for partners is at the heart of gendered family responsibilities.

Among the young and middle aged adults, where variability in health is low, little is known about new family and partnership forms, their relationship with health, and the pathways through which they act. The young population is heterogonous in terms of their ethnic background, and little is known whether the relationship between family forms and health is universal in a society or dependent on migration background and the respective values and norms. And little is known to what extent advantaged and disadvantaged family forms are universal in different welfare state regimes or whether they depend on the social and cultural context of a society.

Among the old, when different ageing trajectories result in an increasing variability in individual health, the health of the partner becomes even more important; in combination with an individual's own health there might be strong mutual influences. Thus, the present family situation and abrupt changes may become more important for an individual's health when compared to the lifelong family biography. Not much is known about if or how partners advancing in age start to resemble each other more closely in terms of health and how the health of the partner influences an individual's own health. Little is known about how geographical and emotional closeness or distance of family members affects health.

Attention to the importance of nature and human health linkages has increased in the past ten years, both in science and in policy [2]. This relates to health benefits from nature-based health-care solutions, such as reducing stress, improving children's immune systems, and reducing the impact from environmental pollution or climate change. This also relates to health risks, such as pollen allergies or infectious diseases transmitted by ticks and mosquitoes. While

Copyright (C) 2021 The Author(s): This is an open-access article distributed under the terms of the Creative Commons Attribution 4.0 International License (CC BY-NC 4.0) which permits unrestricted use, distribution, and reproduction in any medium for non-commercial use provided the original author and source are credited. 
knowledge about and recognition of the importance of nature and human health linkages are increasing rapidly, challenges still remain. Among them are building bridges between relevant, but often still rather disconnected, sectors and topics. There is a need to connect researchers in the fields of health sciences, ecology, social sciences, sustainability sciences and other interdisciplinary sciences, as well as for cooperation with governments, companies and citizens. This need is expressed by both health and nature sectors, and is considered crucial by many for facilitating integrated and practice-oriented approaches.

\section{Medicaid in USA}

Since its original enactment, Medicaid has expanded to cover more uninsured Americans than any other insurance program [3]. After 1996, Medicaid enrollees no longer were required to receive welfare support in order to be a Medicaid beneficiary. Medicaid essentially serves as a broad net that covers those Americans living close to the federal poverty level who are not covered by private insurance plans or Medicare. However, some benefi ciaries do jointly have both Medicare and Medicaid. These beneficiaries are known as "dual eligible". Medicaid provides extra assistance for long-term services excluded under Medicare and further helps these citizens cover Medicare premium costs. Nearly $20 \%$ of all Medicare recipients are dual-enrolled in Medicaid. Medicaid also covers seniors and nursing home residents, lowincome children with working or unemployed parents, a wide range of disabled Americans with mental and physical health conditions, and Americans requiring long-term care. Medicaid is currently the nation's largest source of long-term care funding.

Furthermore, Medicaid serves an expanded role during economic recessions. As more Americans lose jobs and occupation-related health insurance during recessions, they subsequently become eligible for enrollment within Medicaid. The program has no enrollment caps or waiting lists and can therefore absorb more Americans at any given time. According to a report formulated for the Kaiser Foundation, nearly one million more Americans are eligible for Medicaid for every $1 \%$ increase in the nation's unemployment rate. This cyclical nature of Medicaid enrollment consequently places a strain on public hospital systems. As a result, Medicaid is currently accountable for nearly $33 \%$ of public hospital revenues and nearly $40 \%$ of public health center operating revenues at any given time.

Medicaid is a joint program between the federal government and the state governments. The federal government, through its Centers for Medicare and Medicaid Services (CMS) agency under the US Department of Health and Human Services, authorizes each state-sponsored Medicaid program. States in turn have the responsibility of implementing and administrating Medicaid. CMS specifically sets guidelines and regulations, recommends policy changes, and implements amendments to state-level plans. However, the states have the ultimate responsibly of drafting a Medicaid plan that details eligibility categories and requirements, benefi ts that will be covered for Medicaid recipients, and how the Medicaid program will be administered in each state, as long as these guidelines fall within the minimum guidelines established by the CMS. States are also responsible for maintaining transparency by providing readily accessible information about the rules, policies, eligibility requirements, and benefits received for potential Medicaid beneficiaries. Furthermore, each state is required to run a "medical care advisory committee" which oversees the development of new policies and any changes in Medicaid administration on the state level.

\section{Health Care in UK}

The NHS and Community Care Act 1990 is seen by many as a turning point in the provision of care for the elderly [4]. We are witnessing changes fuelled by the legislation that also created health care Trusts. Trusts are responsible for the direct provision of care to patients who have nursing needs. Social services departments, on the other hand, have responsibility for the provision of care to individuals who have social needs. Private concerns are also involved as providers of care for the elderly in the community by complementing health and social services; they may be funded by government sources. Care is 'purchased' from the providers by the health authorities or boards through a system known as internal marketing. The authorities or boards are expected by law to provide community health services in order to ensure the patient's continued independence in the community. Sometimes, however, problems can arise over whether a health authority/board or a local authority (the local council) should pay for clients whose health and social needs overlap.

Care of the elderly and how it is best achieved can highlight emotive issues, especially where there appears to be a deficit between clients' needs and what can be realistically provided for them. This should nevertheless be seen against a background of an increasingly healthy ageing population that is competing for limited and diminishing resources with other client groups. While it is true that the law is sometimes used by governmental agencies as a framework within which care must be provided, it is also true that there is a link between government policies and the actual level of care provided. Emphasis on care in the community has seen an argument develop over rationalisation because of the limited resources being put forward by Trusts when hospital closures and centralisation plans are implemented. Where there has been a reduction in services, recent court decisions have shown that the courts are prepared to support what they perceive 
as 'reasonable' measures by Trusts and social services departments. The Trusts and social services must provide the best albeit limited - care that they can in the circumstances when available resources are taken into account.

\section{Chronic Diseases}

Longevity and lifestyle choices such as smoking, alcohol, and obesity have contributed to people developing more chronic illnesses [5]. The occurrence of multiple chronic conditions increases with age, which compounds the burden of caring for the growing aging population. Almost one half of older adults in America are living with both chronic conditions and functional limitations. Eighty percent have at least one chronic condition, and 50 percent have at least two. Approximately 75 percent of Americans 65 and older are living with multiple chronic conditions and 20 percent are living with five or more chronic conditions. The oldest old population (80 and older) is growing most rapidly and has the highest rates of comorbidity.

It is more expensive to meet the complex care needs of people with multiple chronic conditions. Many will also need supportive help because those with multiple chronic conditions experience higher levels of poor functional status. Older adults who are living with five or more chronic illnesses have, on average, 50 prescriptions and 14 different physicians and make 37 office visits annually. Those with multiple chronic conditions account for 71 percent of the total healthcare spending in the United States. The fee for service individuals with multiple chronic conditions, who are beneficiaries of the government-sponsored Medicare, accounts for 93 percent of the total Medicare spending. The unsustainability of medical costs is an incentive for the Centers for Medicare and Medicaid to support more efficient, less costly, and better quality systems of care for the sickest people. The financial burden is also borne by people living with multiple chronic conditions through out of pocket costs and the high price of prescription medications.

Meeting the healthcare and social needs of the older population is a worldwide public health challenge. To properly and sustainably meet the needs of older adults, providers must challenge fragmented and complex care and social support systems and implement coordinated, person-centered care across a variety of care settings and providers. Providers must also foster chronic disease self-management programs and other forms of patient engagement. Two important concepts that we address throughout the book that serve to promote higher-quality accessible care with greater patient satisfaction at a lower cost are person-centered and value-based care.

\section{Insurance}

The purchase of insurance is a rational decision by consumer/patients to ensure they can access and afford medical care [6]. It is a rational way for third-party insurance companies to make money. And it is a rational way for providers to ensure and increase their business. Insurance is rationally advantageous for these three main entities involved in private medical care. This is because it "insures" against two critical problems in the market for health: uncertainty and asymmetric information.

Health, as well as health care, is intrinsically uncertain ; properly dealing with uncertainty is, in many ways, a critical component in becoming a talented medical professional. Both the patient and the provider have little or no idea when sickness will strike. Therefore, it is diffi cult for the patient to plan for health-care access ahead of time. In the event that the ill can afford the proper care, there is no guarantee for ideal, certain outcomes from that treatment. Medicine is one of the most scientific disciplines, but does not always have predictable outcomes. The uncertainty about when and if the patient/consumer will need care, plus the uncertainty about the effectiveness and value of care, means that the health-care market is far from efficient.

The provision of health care also has intrinsic information asymmetries. Both providers and patients possess their own knowledge, which is often unavailable to the other party. Physicians have substantially more knowledge about health conditions than do patients, and they often charge for this expertise as a commodity. Sometimes doctors even "decommodify" themselves, saying we are "the best at joint replacements" or "we have the lowest infection rate" or "we will see you the fastest" - claims that patients might not completely understand. It is also difficult for doctors and insurers to decide on an upfront cost for care, as patients might withhold information about comorbidities or medical history. With this gap in knowledge and ambiguity in market price, there is a deadweight loss in market efficiency; there is a less-than-optimal provision of medical care.

Health uncertainty means that providers may not be paid for treatment except when disaster strikes, and at that time patients may not be able to pay for the expensive care. Here, at the point of service, supply and demand might not match up and purchased health care is foregone. In addition, patients do not possess "full and relevant information" about what treatment they require (as the doctors do), and they might withhold information from the doctor about their illness or ability to pay. Consequently, lack of information means that doctors are less likely to provide services and patients are less likely to seek it. 


\section{Prevention}

Preventive measures in health care have been discussed as a means of avoiding disease or providing early detection for improvement in health with a concurrent minimization of health care expenditures [7]. Inherently, it initially makes sense that, if a disease can be detected early or prevented altogether, the cost of treating it can be reduced or eliminated and overall health care spending should decrease. Yet, this has not been borne out, and debate about potential savings and value of clinical preventive services has become more polarized. Some preventive services can reduce health care costs, but many do not, and others may actually increase health care costs over a lifetime. In a review of the costeffectiveness of selected clinical preventive services, the evidence does not support the idea that global prevention reduces medical spending, and the vast majority of other clinical preventive services do not save money. In fact, overall costs to the health care system typically go up when disease-preventing strategies are put into practice.

Despite potential overall increased costs, some experts have suggested that clinical preventive services are still worthwhile when they provide good value, defined as substantial health benefit per dollar spent net of any savings. Applying this concept, many preventive services are cost-effective, even when they do not reduce lifetime total cost. The National Commission on Prevention Priorities reviewed recommended preventive services known to improve health and found that 16 of them increased costs while only five services decreased cost. Only a limited number of services have since been shown to decrease costs and enhance life-years saved: childhood immunization series, smoking cessation advice and assistance, discussion of daily aspirin use to prevent cardiovascular disease, and breast and colorectal cancer screening. Today, research continues to evaluate not only the value of disease prevention and health promotion efforts but also the most effective manner of dissemination and adoption of recommendations. It has been shown that combining targeted campaigns to increase access to preventive services with more comprehensive community programs may yield even greater cost savings.

\section{Clinical Education}

Clinical education in the 21 st century will also need to take a broader view of medicine and health, with greater emphasis on understanding the social, behavioral, cultural, and environmental factors that influence health and disease in addition to understanding the biological basis of disease [8]. Developing this understanding will in turn require that biomedical science be better integrated with a patient- and population based approach that addresses the determinants of disease and health, and places greater emphasis on prevention and the identification of risk factors and how to mitigate them.

The focus on the biomedical basis of disease that characterizes the current model for clinical education assumes that ill health is fully explained by disease, so that the core of medical science is the diagnosis and treatment of disease. American medicine, however, is being asked to move beyond this model to address issues related to population health, resource allocation, new means for caring for chronic disease, and the management of health information, all areas in which physicians have traditionally not been trained. Medical schools in particular are believed to produce physicians well equipped to deal with specific organ systems or pathologies, but ill equipped to deal with the behavioral causes of chronic diseases or the social context of illness. According to one survey of young physicians, fewer than half reported receiving excellent or good preparation in coordinating patient care with community services, providing cost-effective care, or managing the needs of the frail elderly. Nursing tends to be more oriented toward health promotion and disease prevention. Advanced practice nurses in particular are focused on establishing knowledge partnerships with their patients, educating them about their conditions, and engaging them in illness prevention and health promotion.

\section{Practice}

As a consequence of modern medicine and modern style of living, two demographic trends, namely longevity and a decline in fertility have greatly increased the aging population [9]. The number of older persons aged 60 years or over is expected to be 1.4 billion by 2030 (World Population Data 2017). This demographic change combined with changes in family structure challenges the future of elderly care, and contributes to grounding a case towards the use of advanced robotics and AI to either integrate or radically replace human-provided services in this field.

Traditionally, the practice of care-in particular for the elderly-was rooted among family members. In traditional proximate families, the exchange of care is constituted by ritualized face-to-face conversations, bodily contact and daily interactions among members characterized by physical co-presence. However, massive social changes caused a progressive disruption of the "traditional" extended family, and income inequalities ever more induce young generations to be on the move uprooting themselves geographically, culturally, and economically to find a better life.

In contemporary societies, institutional care is a substitute for family-oriented patterns of care, in order to provide care for the elderly. While care for older people through non-member family caregivers can be morally understandable, it is important to give the elderly a sense of belonging, regard, and comfort in the vulnerable stage of 
their lives. The virtue of acknowledged dependence is the commitment of the community to address the needs, values, and best interest of the elderly in providing care for them.

\section{CONCLUSION}

Primary health care services are provided by family physicians, gynecologists, pediatricians, dentists and health visitors. Primary care teams are made up of a doctor and a nurse, and primary care physicians are most often the people with whom patients make first contact with the health care system. A referral is not required to go to a primary care doctor, but in most cases it is necessary to order, ie arrange an arrival date in advance. In the event that a person's health condition requires specialist health care in terms of examinations, certain diagnostic tests and/or treatment, the primary care doctor will refer the person to an appropriate specialist doctor by issuing an appropriate referral. The patient should get an appointment in advance from the health institution to which he was referred. Secondary levels of health care include hospitals, clinics, and special rehabilitation hospitals. At the tertiary level, there are clinical hospitals and clinical hospital centers. In addition to providing the most comprehensive health care, tertiary-level institutions are also in charge of medical education and research.

\section{REFERENCES}

1. Doblhammer, G., \& Gumà, J. (2018). „Summary and Research Implications“ in Doblhammer, G., \& Gumà, J. (eds): „A Demographic Perspective on Gender, Family and Health in Europe“, Springer International Publishing AG, Cham, Switzerland, pp. 9-10.

2. Keune, H.; Friesenbichler, K.; Häsler, B.; Hilgers, A.; Jäppinen, J. P.; Job-Hoben, B.; Livoreil, B.; Oosterbroek, B.; Romanelli, C.; Soubelet, H.; Stadler, J.; Ströher, H.; Tapaninen, M. (2019.): „European Nature and Health Network Initiatives“ in Marselle, M. R.; Stadler, J.; Korn, H.; Irvine, K. N.; Bonn, A. (eds): „Biodiversity and Health in the Face of Climate Change“, Springer Nature Switzerland AG, Cham, Switzerland, pp. 331.

3. Sathiyakumar, V., Apfeld, J. C., \& Sethi, M. K. (2013). „Medicaid and the State Children's Health Insurance Program“ in Sethi, M. K., \& Frist, W. H. (eds). „An Introduction to Health Policy - A Primer for Physicians and Medical Students“, Springer Science+Business Media, New York, USA, pp. 29.

4. Fletcher, L., \& Buka, P. (1999). „A Legal Framework for Caring - An Introduction To Law And Ethics In Health Care“, Macmillan Press Ltd, Basingstoke, UK, pp. 147-148.

5. Galiana, J., \& Haseltine, W. A. (2019). „Aging Well - Solutions to the Most Pressing Global Challenges of Aging“, Palgrave Macmillan, Springer Nature Singapore Pte Ltd., Singapore, Singapore, pp. 9-10.

6. Rosman, D. A., \& Apfeld, J. C. (2013). „The Economics of Health Care“ in Sethi, M. K.; \& Frist, W. H. (eds): „An Introduction to Health Policy - A Primer for Physicians and Medical Students“, Springer Science+Business Media, New York, USA, pp. 135.

7. Smith, H. A. (2013). „Prevention and US Health Care“ in Sethi, M. K., Frist, W. H. (eds): „An Introduction to Health Policy - A Primer for Physicians and Medical Students“, Springer Science+Business Media, New York, USA, pp. 75.

8. Kohn, L. T. (ed) (2004). „Academic Health Centers - Leading Change in the 21st Century“, National Academies Press, Washington, USA, pp. 48-49.

9. Bertolini, A., \& Arian, S. (2020).,, Do robots care? Towards an anthropocentric framework in the caring of frail individuals through assistive technology“ in Haltaufderheide, J., Hovemann, J., \& Vollmann, J. (eds): „Aging between Participation and Simulation - Ethical Dimensions of Social Assistive Technologies“, Walter de Gruyter GmbH, Berlin, Germany, pp. 36-39.

CITATION: Siniša Franjić (2021). Health Care for Elderly. South Asian Res J Nurs Health Care, 3(2): 17-21. 